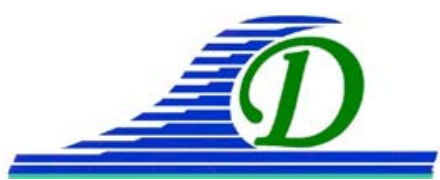

XIII İ̀es Journées Nationales Génie Côtier - Génie Civil

Dunkerque, 2-4 juillet 2014

DOI:10.5150/jngcgc.2014.057 C Editions Paralia CFL

disponible en ligne - http://www.paralia.fr-available online

\title{
La mesure du niveau de la mer par bouées GPS
}

\section{Gaël ANDRE ${ }^{1}$, Belén MARTÍN MÍGUEZ ${ }^{2}$, Valérie BALLU ${ }^{3,5}$, Laurent TESTUT ${ }^{4}$, Guy WÖPPELMANN ${ }^{5}$}

1. Service hydrographique et océanographique de la Marine (SHOM), Brest, France. gael.andre@shom.fr

2. Centro Tecnológico del Mar (CETMAR), Vigo, Espagne. bmartin@cetmar.org

3. Institut de Physique du Globe de Paris (IPGP), La Rochelle, France. valerie.ballu@univ-lr.fr

4. LEGOS (CNES-IRD-CNRS-Université Paul Sabatier), Toulouse, France. laurent.testut@legos.obs-mip.fr

5. LIENSs (Université de la Rochelle - CNRS), La Rochelle, France. guy.woppelmann@univ-lr.fr

\section{Résumé :}

La mesure du niveau de la mer dans un référentiel mondial avec une précision subcentimétrique représente un réel défi dans le contexte actuel du réchauffement climatique et de l'élévation du niveau des mers qui en résulte. Les systèmes mondiaux de navigation par satellite GNSS (Global Navigation Satellite System) permettent la mesure directe du niveau de la mer rapporté à un référentiel géocentrique absolu. Nous présentons ici les résultats d'une expérience multi-instruments avec trois bouées équipées d'un système de positionnement par satellite GPS (Global Positioning System), un marégraphe radar et une échelle de marée. Cette expérience s'est déroulée à l'île d'Aix (côte ouest de la France) les 27 et 28 mars 2012. L'erreur quadratique moyenne (RMSE) calculée à partir de la différence entre les données des bouées GPS et celles du marégraphe radar est comprise entre $1 \mathrm{~cm}$ et $2,2 \mathrm{~cm}$, ce qui est satisfaisant pour les applications marégraphiques et offre d'intéressantes perspectives pour les futures études sur l'évolution du niveau de la mer.

Mots-clés : Bouées GNSS, Marégraphe radar, Hauteur d'eau, Ellipsoïde.

\section{Introduction}

En dépit des avancées technologiques récentes de l'altimétrie satellitaire, les mesures du niveau de la mer destinées aux applications marégraphiques et aux études sur les variations du niveau de la mer liées au changement climatique reposent encore essentiellement sur les mesures locales effectuées par les marégraphes. La principale limite des marégraphes conventionnels réside dans leur ancrage au sol et leur exposition aux mouvements terrestres. La technique GNSS présente l'avantage de fournir des données directement rapportées à un système de référence géocentrique mondial alors 
que les marégraphes fournissent des mesures du niveau de la mer par rapport à la croûte terrestre. Différentes applications des bouées GPS ont déjà été publiées, telles que l'étalonnage des marégraphes côtiers sur des îles éloignées (WATSON et al., 2008 ; TESTUT et al., 2010 ; MARTÍN-MÍGUEZ et al., 2012 ; FUND et al., 2013) et dans des zones au large (BOUIN et al., 2009 ; BALLU et al., 2010).

Ces précédents travaux ont encouragé trois équipes françaises différentes, le Service Hydrographique et Océanographique de la Marine (SHOM), l'Institut National des Sciences de l'Univers (INSU) et l'Institut de Physique du Globe de Paris (IPGP), à concevoir leurs propres bouées GPS. En 2012, ces équipes ont mené une expérience d'inter-calibration sur l'île d'Aix sur la côte ouest de la France (figure 1). L'île d'Aix est un observatoire historique du niveau de la mer équipé d'un marégraphe radar, d'un marégraphe à pression, d'une station météorologique et d'une station GPS permanente. Par ailleurs, les autorités locales sont sensibilisées aux questions scientifiques liées à l'élévation du niveau de la mer et aux risques côtiers, tout particulièrement depuis le passage de la tempête Xynthia ayant sévèrement frappé cette partie des côtes les 27 et 28 février 2010.

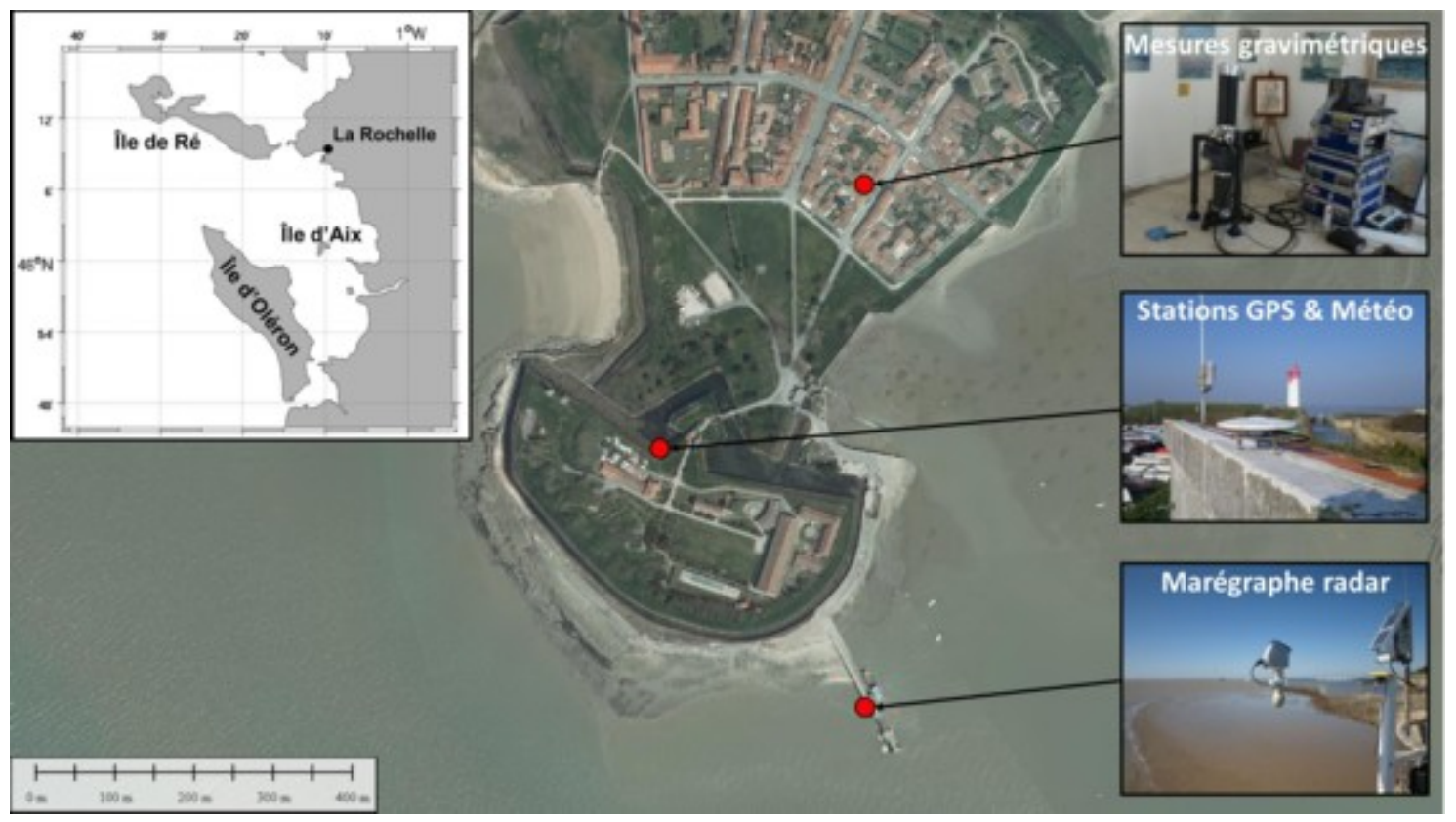

Figure 1. Observatoire du niveau de la mer de l'île d'Aix. Cet observatoire comprend un marégraphe radar, une station météorologique et une station GPS (Photos : SHOM).

\section{Matériel et méthodes}

\subsection{L'expérience}

Les 27 et 28 mars 2012, trois prototypes de bouées GPS (figure 2) ont été déployés à proximité de l'île d'Aix. Les principales caractéristiques des différentes bouées GPS 


\section{XIII $I^{\text {èmes }}$ Journées Nationales Génie Côtier - Génie Civil \\ Dunkerque, 2-4 juillet 2014}

sont fournies dans le tableau 1. Le site de déploiement est une zone située à environ 100 $\mathrm{m}$ du quai (figure 3) où sont installés l'échelle de marée, le marégraphe radar et le marégraphe à pression appartenant au réseau SHOM-RONIM (Réseau d'Observation du Niveau de la Mer). La zone d'expérimentation est également située à proximité de la station de référence ILDX du réseau GNSS permanent (RGP) de l'IGN (Institut Géographique National). Le 28 mars, des mesures de hauteur d'eau ont été prises manuellement sur un cycle de marée par lecture directe de l'échelle de marée et avec une sonde lumineuse. Par ailleurs, des mesures de gravité absolue et relative, des courants océaniques et de nivellement, ont été réalisées.
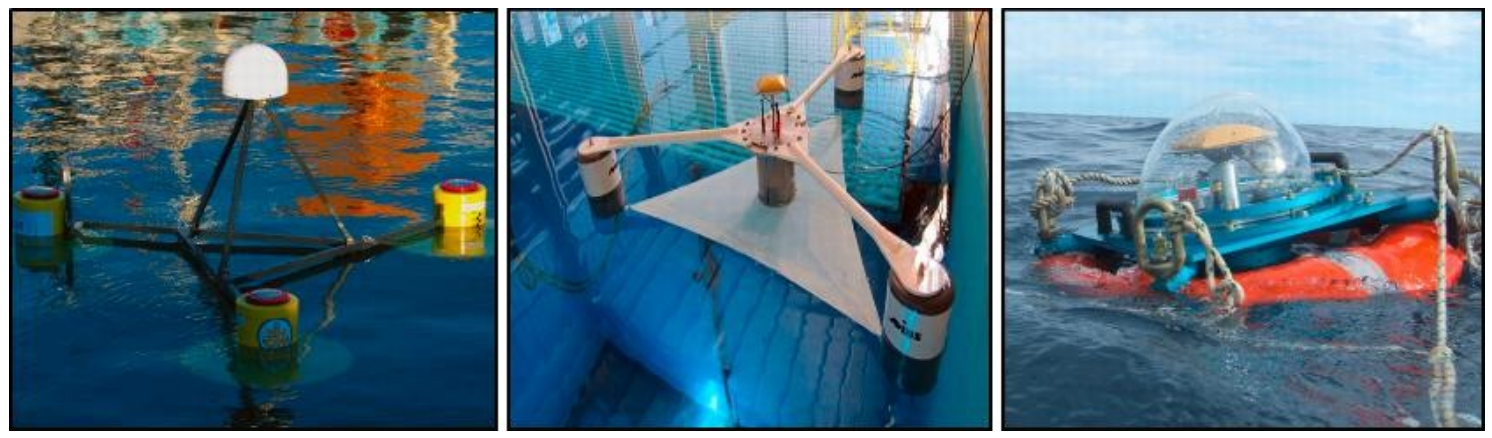

Figure 2. Les trois bouées GPS. SHOM (à gauche), INSU (au centre) et IPGP (à droite)

(Photos : SHOM ; INSU).

Tableau 1. Dimensions et caractéristiques principales des bouées.

\begin{tabular}{lcclcll}
\hline Bouée & Poids & Diamètre & $\begin{array}{l}\text { Hauteur } \\
\text { d'antenne }\end{array}$ & Autonomie & Récepteur & Antenne \\
\hline SHOM & $65 \mathrm{~kg}$ & $2.5 \mathrm{~m}$ & $92.7 \mathrm{~cm}$ & 10 jours & $\begin{array}{l}\text { Trimble SPS852 } \\
\text { GNSS }\end{array}$ & $\begin{array}{l}\text { Leica AT504GG } \\
\text { LEIS choke ring }\end{array}$ \\
\hline INSU & $20 \mathrm{~kg}$ & $2 \mathrm{~m}$ & $33.5 \mathrm{~cm}$ & 5 jours & $\begin{array}{l}\text { TOPCON } \\
\text { GB1000 }\end{array}$ & $\begin{array}{l}\text { PG-A1 avec plan de } \\
\text { masse }\end{array}$ \\
\hline IPGP & $30 \mathrm{~kg}$ & $0.75 \mathrm{~m}$ & $14.9 \mathrm{~cm}$ & 3 jours & $\begin{array}{l}\text { TOPCON } \\
\text { GB1000 }\end{array}$ & $\begin{array}{l}\text { PG-A1 avec plan de } \\
\text { masse }\end{array}$ \\
\hline
\end{tabular}

Les données ont été collectées par les trois bouées du 27 au 28 mars pendant environ 30 heures. L'enregistrement de la bouée IPGP a été interrompu le 27 mars entre $13 \mathrm{~h} 28$ et 14h44 en raison d'une entrée d'eau dans le module de réception. Les données GPS ont été enregistrées à $5 \mathrm{~Hz}$ pour les bouées INSU et SHOM et à $1 \mathrm{~Hz}$ pour la bouée IPGP. 


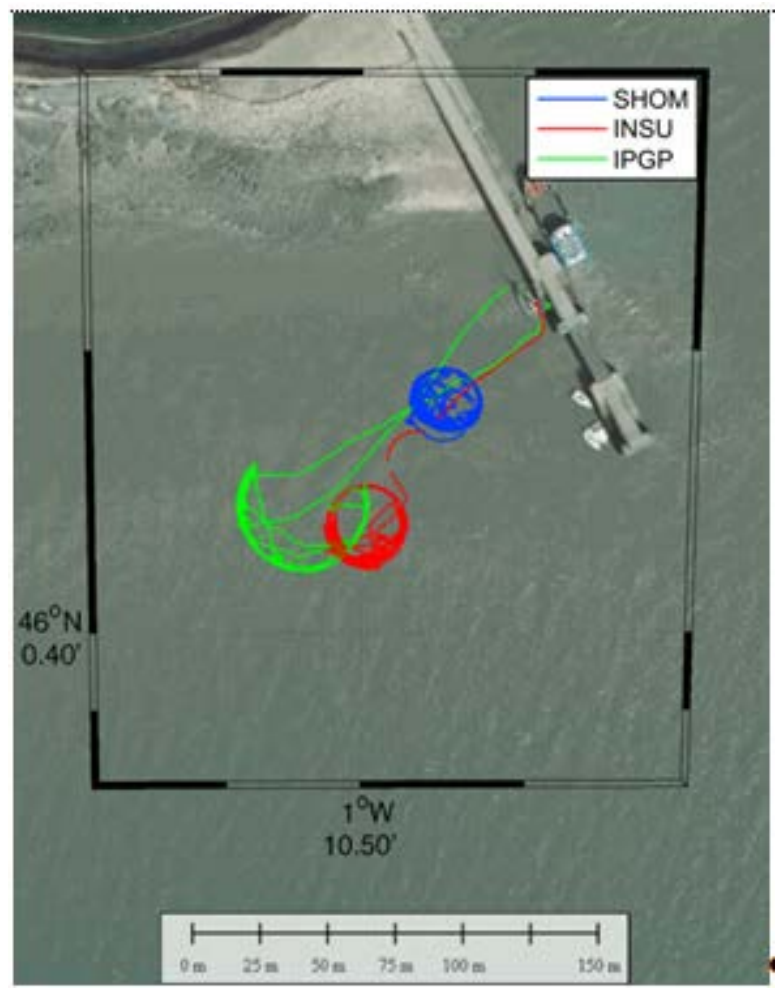

Figure 3. Trajectoires des bouées GPS. Les récepteurs GPS des bouées IPGP et INSU ont commencé à enregistrer alors qu'ils se trouvaient sur le quai, ce qui explique pourquoi le remorquage des bouées par bateau entre le quai et la position du mouillage est visible. En revanche, le récepteur de la bouée SHOM a été branché après le mouillage de la bouée.

\subsection{Traitement des données GPS}

Les données GPS ont été post-traitées en mode cinématique en utilisant la station de référence fixe ILDX. Le traitement a été réalisé en mode différentiel avec résolution entière des ambiguïtés. Ceci implique d'avoir recours à une station de référence statique dans un lieu connu qui collecte les données et qui réduit en même temps les erreurs systématiques communes aux stations cinématiques et fixes, telles que les corrections atmosphériques, d'orbite des satellites, d'horloge des satellites et des récepteurs. La précision de la solution dépend de la longueur de la ligne de base puisque l'utilisation d'une méthode différentielle suppose que l'ionosphère et la troposphère sont identiques aux deux extrémités de la ligne de base. Par conséquent, la méthode de traitement cinématique ne permet pas de traiter avec précision les données des bouées GPS à une trop grande distance d'une station de référence fixe à terre. Les progrès en matière de méthode PPP (Precise Point Positioning) offrent une solution alternative qui permettra de réaliser à l'avenir des mesures à une plus grande distance des côtes (FUND et al., 2013). 


\section{XIII $I^{\text {èmes }}$ Journées Nationales Génie Côtier - Génie Civil \\ Dunkerque, 2-4 juillet 2014}

Le traitement des données s'est fait en deux étapes en utilisant les orbites précises du Service International GNSS (IGS) avec un angle de coupure de $10^{\circ}$ pour toutes les stations. Dans un premier temps, les données de la station à terre ILDX ont été traitées en mode statique à l'aide du logiciel GAMIT/GLOBK (HERRING et al., 2010a et 2010b) afin de calculer sa position dans l'ITRF 2008 (International Terrestrial Reference Frame) (ALTAMIMI et al., 2011), en utilisant l'ellipsoïde du système de référence géodésique GRS80. Dans un second temps, les positions des bouées ont été estimées par rapport à cette station ILDX à l'aide du module TRACK de la suite logicielle GAMIT/GLOBK permettant le traitement cinématique en mode différentiel. Dans notre cas, la station de référence à terre ILDX est très proche de la zone de déploiement des bouées GPS (environ 400 mètres).

\section{Résultats}

\subsection{Trajectoires des bouées}

Les positions horizontales des trois bouées ont été calculées à l'aide du logiciel TRACK. Les trajectoires suivies par les bouées sont représentées sur la figure 3. Les bouées GPS ont été mouillées entre 50 mètres (SHOM) et 100 mètres (IPGP et INSU) du quai sur lequel le marégraphe radar est installé. Les bouées sont fixées à un poids mort immergé autour duquel elles peuvent bouger librement jusqu'à une distance maximale imposée par la longueur de l'amarre. Les trois bouées ayant été déployées sur plus d'une journée, elles ont été soumises aux courants des marées, ce qui explique la forme quasi-circulaire observée. Dans cette zone, les courants sont forts et localement très variables en raison de la présence du quai. On peut noter que la bouée IPGP n'a pas décrit un cercle complet et que la tension sur l'amarre semble plus constante (figure 3). Ceci est probablement dû à une position différente de cette bouée par rapport aux courants locaux et indique que les caractéristiques du flux hydrodynamique peuvent fortement varier localement.

\subsection{Résultats de la comparaison}

La figure 4 montre les hauteurs du niveau marin mesurées par le marégraphe radar $(\mathrm{He})$ par rapport à l'ellipsoïde GRS80 et les séries temporelles des différences $(\Delta \mathrm{He})$ entre les hauteurs du niveau marin des trois bouées GPS et celles du marégraphe radar. Ces résultats montrent une bonne concordance entre les données des bouées GPS et celles du marégraphe. Les valeurs statistiques ont été calculées à partir des différences pour les trois bouées. La différence moyenne est comprise entre $0,13 \mathrm{~cm}$ et $1,84 \mathrm{~cm}$, l'écart type entre 0,94 et 2,15 cm et l'erreur quadratique moyenne (RMSE) entre 0,95 et $2,18 \mathrm{~cm}$ (voir tableau 2). 


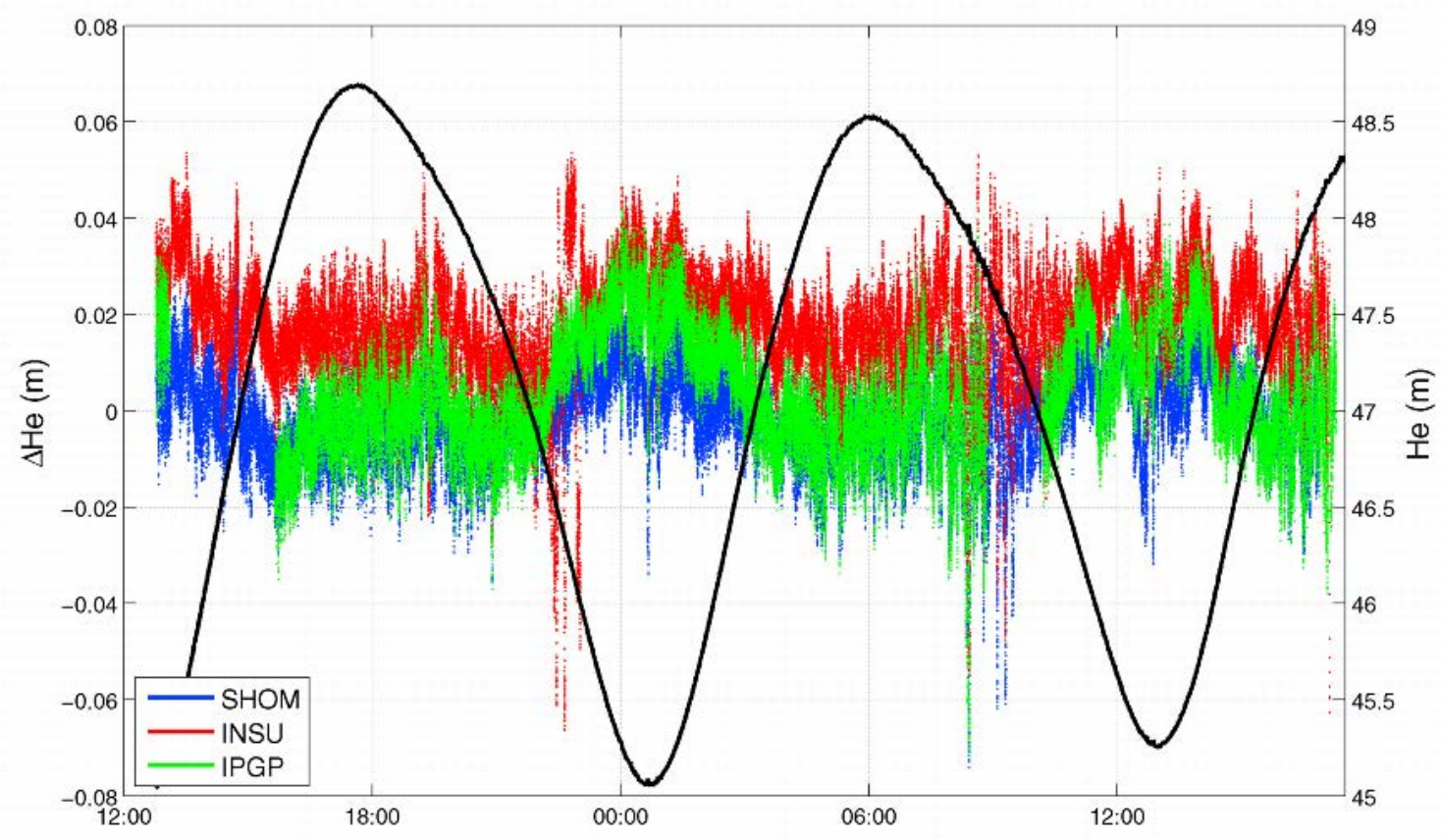

Figure 4. Les hauteurs du niveau marin mesurées par le marégraphe radar (He) audessus de l'ellipsoïde GRS80 sont indiquées en noir (axe Y de droite), les différences ( $\triangle H$ He) entre les séries temporelles de hauteurs des trois bouées GPS et les hauteurs du niveau marin prises par le marégraphe radar (He) sont illustrées en couleur (axe Y de gauche).

Les différences entre les statistiques des trois bouées reflètent différents phénomènes :

a) la valeur moyenne des différences correspond à la précision du référencement "absolu" intégrant les biais de traitement GPS, les erreurs de nivellement résultant à la fois de la détermination de la hauteur de l'antenne par rapport à la surface de l'eau et du référencement du marégraphe par rapport à l'ellipsoïde (nivellement entre le marégraphe et la station de référence ILDX), et également la différence locale de hauteur d'eau entre le marégraphe de référence (à savoir le marégraphe radar) et la bouée GPS résultant de la topographie dynamique locale ;

b) l'écart type reflète le niveau de bruit de chaque série temporelle intégrant, d'une part, le mouvement réel de la bouée et, d'autre part, le bruit aléatoire de traitement, du radar et des instruments GPS. Comme le prévoit sa conception, la bouée SHOM est la plus stable : elle filtre mécaniquement le mouvement induit par les courtes ondes de surface. En revanche, la bouée IPGP est plus sensible aux ondes haute fréquence ;

c) la RMSE est l'association des deux facteurs décrit ci-dessus. Elle reflète dans un seul paramètre le bruit de la série (y compris le mouvement réel de la bouée) et la différence au niveau du référencement absolu. 


\section{XIII $I^{\text {èmes }}$ Journées Nationales Génie Côtier - Génie Civil \\ Dunkerque, 2-4 juillet 2014}

Tableau 2. Valeurs statistiques obtenues par les séries temporelles des différences entre les données des bouées GPS et celles du marégraphe radar.

\begin{tabular}{llll}
\hline Bouée & Moyenne & Ecart-type & RMSE \\
\hline SHOM & $0.13 \mathrm{~cm}$ & $0.94 \mathrm{~cm}$ & $0.95 \mathrm{~cm}$ \\
$I N S U$ & $1.84 \mathrm{~cm}$ & $1.14 \mathrm{~cm}$ & $2.16 \mathrm{~cm}$ \\
$I P G P$ & $0.33 \mathrm{~cm}$ & $2.15 \mathrm{~cm}$ & $2.18 \mathrm{~cm}$ \\
\hline
\end{tabular}

\section{Conclusions}

L'expérience multi-appareils menée sur l'île d'Aix (côte ouest de la France) les 27 et 28 mars 2012 est un cas pratique permettant d'évaluer les performances de plusieurs bouées GPS pour la mesure précise du niveau de la mer. Ainsi, trois bouées équipées de GPS, conçues par trois organismes indépendants (SHOM, INSU et IPGP), ont été déployées. La comparaison entre les mesures fournies par les bouées et le marégraphe radar a clairement démontré les performances satisfaisantes des bouées. Ces résultats indiquent que les bouées GPS sont capables de mesurer la hauteur du niveau marin au centimètre près, un degré de précision comparable à celui du marégraphe radar de référence. Ceci prouve le potentiel global dont disposent ces bouées pour mesurer avec précision les hauteurs du niveau marin directement référencées à l'ellipsoïde GRS80.

Les mesures du marégraphe conventionnel sont par nature liées à la terre. Par conséquent, elles sont limitées aux zones côtières et leur pertinence dans les études globales dépend de la qualité du suivi des mouvements terrestres. Les mesures des bouées GPS représentent un outil prometteur pour étalonner les marégraphes et réaliser des mouillages côtiers, et permet de les référencer directement à un système de référence géocentrique absolu puisqu'elles ne dépendent pas des mouvements terrestres. Néanmoins, cet outil présente encore certaines contraintes pratiques, à la fois au niveau technique (autonomie de la batterie et robustesse de la conception pour une résistance en haute mer) et du traitement des données.

De nouvelles perspectives de traitement des données pour les zones off-shore sont offertes par le développement des techniques PPP. Plusieurs études ont déjà utilisé cette méthode de traitement afin de déterminer les positions des bouées (KUO et al., 2012 ; FUND et al., 2013). Les résultats ne sont pas encore aussi concluants que ceux obtenus avec le traitement différentiel lorsqu'une station de référence proche est disponible. Cependant, grâce aux améliorations continues des méthodes de traitement PPP, l'utilisation des bouées GPS ne devrait plus être très longtemps limitée aux zones côtières.

\section{Remerciements}

Les auteurs remercient les autorités locales de l'île d'Aix pour l'intérêt qu'elles ont porté et leur soutien logistique. Les données de hauteur d'eau de l'île d'Aix sont la propriété 
Thème 3 - Instrumentation, mesures, imagerie et télédétection

du LIENSs, du SHOM et du CG17 et sont disponibles sur le site web REFMAR (http://refmar.shom.fr). Cette expérience a été menée dans le cadre du projet SONEL (Système d'Observation du Niveau des Eaux Littorales) (www.sonel.org).

\section{Références bibliographiques}

ALTAMIMI Z., COLLILIEUX X., METIVIER L. (2011). ITRF2008: an improved solution of the International Terrestrial Reference Frame. Journal of Geodesy, Vol. 85(8), pp 457-473. http://dx.doi.org/10.1007/s00190-011-0444-4

BALLU V., BOUIN M.N., CALMANT S., FOLCHER E., BORE J.M., AMMANN J., POT O., DIAMENT M., PELLETIER B. (2010). Absolute seafloor vertical positioning using combined pressure gange and kinematic GPS data. Journal of Geodesy, Vol. 84(1), pp 65-77. http://dx.doi.org/10.1007/s00190-009-0345-y

BOUIN M.N., BALLU V., CALMANT S., PELLETIER B., AMMANN J., BORE J.M, FOLCHER E. (2009). Methodology of kinematic GPS experiment for local sea surface mapping, Vanuatu. Journal of Geodesy, Vol. 83(21), pp 1203-1217. http://dx.doi.org/10.1007/s00190-009-0338-x

FUND F., PEROSANZ F., TESTUT L., LOYER S. (2013). An Integer Precise Point Positioning technique for sea surface observations using a GPS buoy. Advances in Space Research, Vol. 51(8), pp 1311-1322. http://dx.doi.org/10.1016/jasr.2012.09.028

HERRING T.A., KING R.W., McCLUSKY S.C. (2010a). GAMIT: Reference Manual. GPS Analysis at MIT, Release 10.4, Massachusetts Institute of Technology, Cambridge. HERRING T.A., KING R.W., McCLUSKY S.C. (2010b). GLOBK Global Kalman Filter VLBI and GPS analysis program, Release 10.4, Massachusetts Institute of Technology, Cambridge.

KUO C.Y., CHIU K.W., CHIANG K.W., CHENG K.C., LIN L.C., TSENG H.Z., CHU F.Y., LAN W.H., LIN H.T. (2012). High-frequency sea level variations observed by GPS buoys using precise point positioning technique. Terr. Atmos. Ocean. Sci., Vol. 23, pp 209-218. http://dx.doi.org/10.3319/TAO.2011.10.05.01(Oc)

MARTÍN-MÍGUEZ B., TESTUT L., WÖPPELMANN G. (2012). Performance of modern tide gauges: towards mm-level accuracy. Scientia Marina, Vol. 76, pp 221-228. http://dx.doi.org/10.3989/scimar.03618.18A

TESTUT L., MARTÍN-MÍGUEZ B., WÖPPELMANN G., TIPHANEAU P., POUVREAU N., KARPYTCHEV M. (2010). Sea level at Saint Paul Island, southern Indian Ocean, from 1874 to the present. Journal of Geophysical Research, Vol. 115, C12028. http://dx.doi.org/10.1029/2010JC006404

WATSON C., COLEMAN R., HANDSWORTH R. (2008). Coastal tide gange calibration: a case study at Macquarie Island using GPS buoy techniques. Journal of Coastal Research, Vol. 24(4), pp 1071-1079. http://dx.doi.org/10.2112/07-0844.1 